\title{
The relation of the abundance ratios in early-type galaxies with the cluster mass
}

\author{
Conrado Carretero ${ }^{1}$, Alexandre Vazdekis ${ }^{1}$ \\ and John E. Beckman ${ }^{1,2}$ \\ ${ }^{1}$ Instituto de Astrofísica de Canarias, Vía Láctea s/n, 38200 La Laguna, Tenerife, Spain email: \\ cch@iac.es \\ ${ }^{2}$ Consejo Superior de Investigaciones Científicas, Spain
}

\begin{abstract}
We present the values of $\mathrm{CN}$ and $\mathrm{Mg}$ overabundances with respect to Fe, for earlytype galaxies in 8 clusters over a range of richness and morphology. Spectra were taken from the Sloan Digital Sky Survey (SDSS) DR1 spectroscopic database, and from WHT and CAHA observations. Abundances were derived from absorption lines and single burst population models, by comparing galaxy spectra with appropriately broadened synthetic model spectra. We find robust correlations between $[\mathrm{Mg} / \mathrm{CN}]$ and $[\mathrm{CN} / \mathrm{Fe}]$ abundance ratios and cluster X-ray luminosity. No correlation was observed for $[\mathrm{Mg} / \mathrm{Fe}]$. We also see a clear trend with the richness and morphology of the clusters. This behavior is interpreted given varying formation timescales for $\mathrm{CN}, \mathrm{Mg}$ and $\mathrm{Fe}$, combined with a different star formation history in early-type galaxies as a function of their environment. This result supports the scenario in which early-type galaxies in more massive clusters are assembled on shorter timescales than those galaxies within less massive clusters.
\end{abstract}

\section{Introduction}

Stellar populations offer a fossil record of the formation and evolution of galaxies, providing very strong constraints on the principal galaxy formation scenarios. So, a programme to understand stellar populations as a function of the environment will give many answers to the puzzle. Differences in the abundance of $\mathrm{CN}, \mathrm{Mg}$ and $\mathrm{Fe}$, as a function of the environment, have been recently suggested by Sánchez-Blázquez et al. (2003). We explore these differences by studying a large number of galaxy clusters over a range of richness and morphology.

\section{Abundance estimations and results}

To derive mean luminosity-weighted ages and metallicities, we have compared selected absorption line strengths with those predicted by the model of Vazdekis (1999). This model provides flux-calibrated spectra in the optical range at a resolution of $1.8 \AA$ (FWHM) for single-burst stellar populations. The selected absorption indices were $\mathrm{CN}_{2}$, $\mathrm{Mg}_{2}$ and $\mathrm{Fe} 2$ (Worthey et al. 1994).

Plots of the strengths of the selected indices versus $\mathrm{H} \beta$ provide rather orthogonal model grids, allowing us to accurately estimate galaxy mean ages as well as the abundances of these elements. Since the $\mathrm{CN}_{2}$ index is by far dominated by $\mathrm{C}$ and $\mathrm{N}$, the $\mathrm{Mg}_{2}$ index is governed by $\mathrm{Mg}$ and the $\mathrm{Fe} 2$ index by $\mathrm{Fe}$, the obtained metallicities can be approximated to $[\mathrm{CN} / \mathrm{H}],[\mathrm{Mg} / \mathrm{H}]$ and $[\mathrm{Fe} / \mathrm{H}]$ abundances. These values are then used to estimate the abundance ratios $[\mathrm{CN} / \mathrm{Fe}],[\mathrm{Mg} / \mathrm{Fe}]$ and $[\mathrm{Mg} / \mathrm{CN}]$ for each galaxy. Finally, we determined the overabundances of each cluster, as the mean values of the overabundances of all the galaxies within it. Errors were computed as the standard deviation of each sample. 


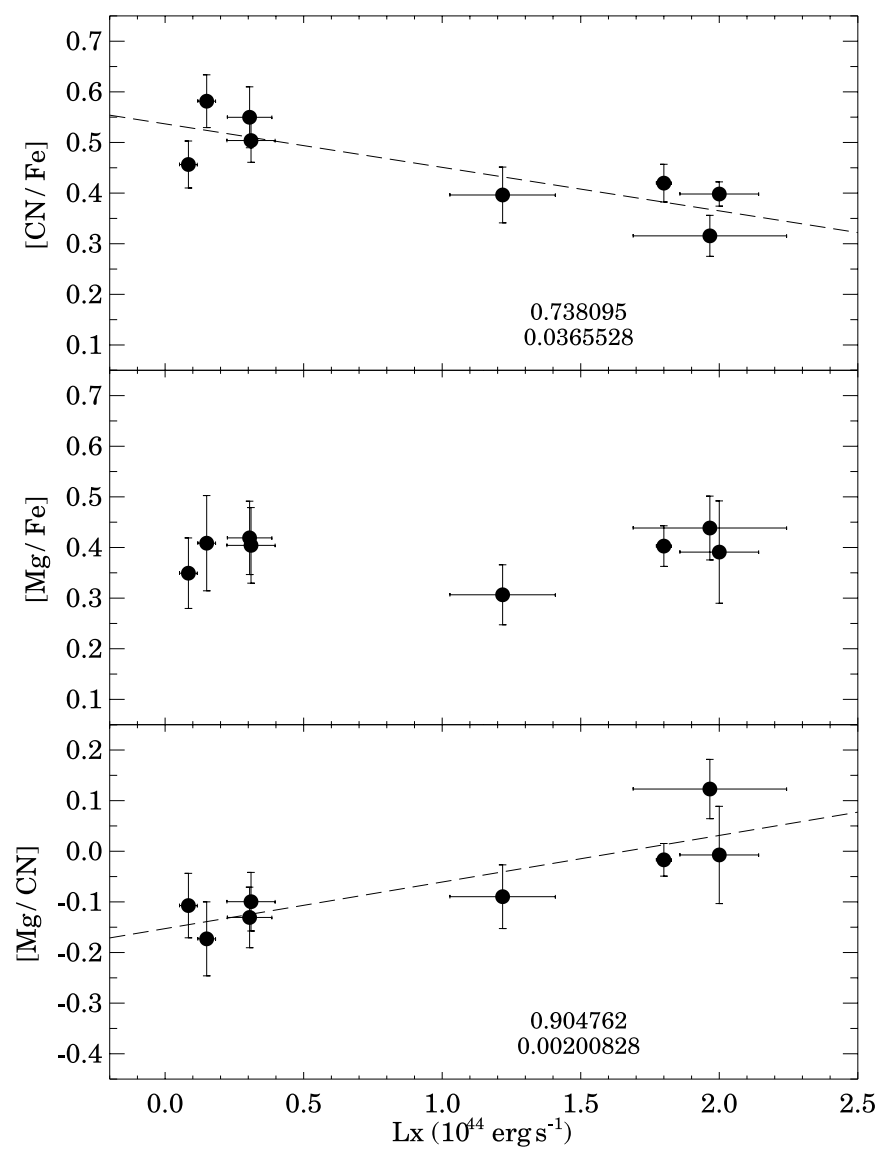

Figure 1. Cluster X-ray luminosity vs. overabundance values of $[\mathrm{CN} / \mathrm{Fe}]$ (top), $[\mathrm{Mg} / \mathrm{Fe}]$ (middle) and $[\mathrm{Mg} / \mathrm{CN}]$ (bottom). Each point corresponds to one individual cluster, and is computed as the mean value of the overabundaces of early-type galaxies within each cluster, with $150 \mathrm{~km} \mathrm{~s}^{-1}<\sigma<250 \mathrm{~km} \mathrm{~s}^{-1}$. The correlation coefficient and its significance are written in top and bottom panels.

We used X-ray luminosity as a quantitative indicator of the mass of the clusters. We adopted X-ray luminosity values from Ebeling et al. (1998) and Ledlow et al. (2003). Figure 1 shows the values of $[\mathrm{CN} / \mathrm{Fe}],[\mathrm{Mg} / \mathrm{Fe}]$ and $[\mathrm{Mg} / \mathrm{CN}]$ versus X-ray luminosity for each cluster. We found clear correlations between $[\mathrm{CN} / \mathrm{Fe}]$ and $[\mathrm{Mg} / \mathrm{CN}]$ values and X-ray luminosity. No correlation was found for $[\mathrm{Mg} / \mathrm{Fe}]$.

\section{Discussion}

The correlations can be interpreted in terms of the different formation timescales for each element, and the different star formation history of early-type galaxies as a function of their environment.

$\mathrm{Mg}$ is ejected into the interstellar medium (ISM) by Type II supernovae (SNe II) on short timescales $(<10 \mathrm{Myr})$. On the other hand, the iron-peak elements are the products of SNe Ia, which occur on timescales of $\sim 1$ Gyr. Between the two extremes, although there are recent suggestions that most of the $\mathrm{C}$ come from massive stars (Akerman et al. 2004), C and N are mainly ejected into the ISM by low- and intermediate-mass stars (e.g. 
Chiappini, Romano, \& Matteucci 2003), leading to CN formation on timescales longer than $\mathrm{Mg}$ but shorter than Fe.

In this framework, the constancy of the $[\mathrm{Mg} / \mathrm{Fe}]$ overabundance values is explained in terms of the great difference in the formation timescales of the two elements: the galaxies are fully assembled before Type Ia SNe can significantly pollute with Fe the ISM of the smaller galaxies before merging, and right after $\mathrm{Mg}$ is fully ejected. Since $[\mathrm{Mg} / \mathrm{Fe}]$ is found to be constant with the X-ray luminosity of the clusters, which is an indicator of their mass, we conclude that this ratio is independent of the environment. Similar results for the $[\mathrm{Mg} / \mathrm{Fe}]$ ratio have been obtained by other authors (e.g. Sánchez-Blázquez et al. 2003), by studying field and Coma cluster elliptical galaxies.

However, when considering species with less disparate formation timescales, such as $\mathrm{CN}$ and $\mathrm{Fe}$, or $\mathrm{CN}$ and $\mathrm{Mg}$, clear correlations are found between abundance ratios and the environment, as shown in Fig. 1. The fact that $[\mathrm{CN} / \mathrm{Fe}]$ decreases with the cluster X-ray luminosity, and that $[\mathrm{Mg} / \mathrm{CN}]$ increases with it, suggest that galaxies in more massive clusters are fully assembled on shorter timescales than those in less massive clusters.

The result that there exist relative differences in the assembly timescales of the galaxies due to the properties of the environment is qualitatively in agreement with the hierarchical models. Discrepancies appear, however, when considering the absolute values of such timescales. The fact that $[\mathrm{CN} / \mathrm{Fe}]$ abundance ratio is not constant implies that early-type galaxies are fully assembled on timescales around the massive release of CN into the ISM. Hierarchical models, on the contrary, predict longer assembly timescales.

\section{Acknowledgements}

We acknowledge support from grant AYA2001-0435 from the Spanish Ministry of Science and Technology.

\section{References}

Akerman, C.J., Carigi, L., Nissen, P.E., Pettini, M., \& Asplund, M. 2004 A\&A 414, 931.

Chiappini, C., Romano, D., \& Matteucci, F. 2003 MNRAS 339, 63.

Ebeling, H., Edge, A.C., Böhringer, H., Allen, S.W., Crawford, C.S., Fabian, A.C., Voges, W., \& Huchra, J.P. 1998 MNRAS 301, 881

Ledlow, M.J., Voges, W., Owen, F.N., \& Burns, J.O. 2003 AJ 126,2740

Sánchez-Blázquez, P., Gorgas, J., Cardiel, N., Cenarro, J., \& González, J.J. 2003 ApJ 590, L91

Vazdekis, A. 1999 ApJ 513, 224

Worthey, G., Faber, S.M., González, J.J., \& Burstein, D. 1994 ApJS 94, 687 